\title{
A pilot study of the body weight of pure-bred client-owned adult cats
}

\author{
Ellen Kienzle* and Katja Moik \\ Animal Nutrition and Dietetics, Ludwig-Maximilians-Universität München, Schönleutner Strasse 8, 85764 Oberschleißheim, \\ Germany \\ (Received 15 October 2010 - Revised 23 February 2011 - Accepted 7 March 2011)
}

\section{Abstract}

A total of 539 pure-bred and seventy-five cats without a pedigree were weighed and scored at cat shows or in veterinary surgeries. Data from normal-weight cats with a body condition score (BCS) of 5 (ideal) were only used. Breeds were grouped into five classes. For female cats, the mean weight for these groups were as follows: very light ( $2.8 \mathrm{~kg})$; light $(3.2 \mathrm{~kg}) ;$ medium $(3.5 \mathrm{~kg}) ; \mathrm{large}(4.0 \mathrm{~kg}) ;$ giant (4.9) kg. For male cats, the corresponding values were $3 \cdot 6,4 \cdot 2,4 \cdot 3,5 \cdot 1$ and $6 \cdot 1 \mathrm{~kg}$. Siamese/Oriental Shorthair were identified as a very light breed, the Norwegian Forest and the Siberian Cat as a large breed and the Maine Coon as a giant breed. Males and females of the same breed did not always belong to the same class. In some breeds, individuals of the same sex were found in two different classes. The percentage of intact overweight cats (BCS > 5) was low (7\% of intact males, $3 \%$ of intact females). Incidence of overweight in neutered cats was $50 \%$ in males and $38 \%$ in females. Among pedigreed cats, there were differences in the incidence of overweight in neutered cats: high in Norwegian Forest Cats (males 75\%, females 50\%) and low in Siamese/Oriental Shorthair Cats (males 25\%, females $1 \%$ ). Cats with a BCS of 6, 7 and 8 had on average 120,154 and $214 \%$ of the normal weight of their breed, respectively.

\section{Key words: Body weight: Overweight: Pure-bred cats}

With regard to the incidence of overweight in pet cats, which has by now reached nearly $50 \%{ }^{(1)}$, prevention of overfeeding is highly important. In addition, although weight loss may reduce energy expenditure in cats, maintaining an ideal weight after weight loss is very difficult ${ }^{(2)}$. For instance, $1 \mathrm{~kg}$ of overweight in a cat may already represent a potential health risk. Therefore, it is very important to have detailed data on the normal weight of cats in order to alert veterinarians and cat owners to the onset and early stages of overweight. Data on the body weight of cats are mostly the data on domestic European Shorthair-type cats without a pedigree. When present, this information is usually included in the Materials and method section of research papers on cats. Often, there is no information on the body condition of the cats. The reader can only guess whether the cats were lean or not. The use of lean cats can be presumed in studies where intact young adult animals are used, in studies with intact cats in a breeding colony ${ }^{(3)}$ or in a breeding experiment $^{(4,5)}$. Leanness of experimental cats is also likely in studies where chemical analyses ${ }^{(6,7)}$ or dual-emission X-ray absorptiometry analysis was carried out and the cats show a reasonable fat content ${ }^{(8)}$. Data on cats of other breeds than domestic non-pedigreed European Shorthair cats are scarce.
In the present study, more data on the relationship between breed, sex, body condition scores (BCS) and body weight in cats were collected.

\section{Materials and methods}

A total of 539 pure-bred and seventy-five adult cats without a pedigree were weighed and scored (BCS by Laflamme ${ }^{(9)}$ ) at cat shows (pure-bred cats, South of Germany and Elsass in France breeders came also from different areas) or in veterinary surgeries (mostly cats without a pedigree, small-town, small-animal practices, without high specialisation) in the South of Germany in a prospective study. Cats were considered to be adult at $\geq 1$ year: 192 were intact males, 247 intact females, 109 neutered males and sixty-six neutered females. With the exception of two Norwegian Forest cats (mother and daughter), there were no direct relatives. The same mobile baby scale was used for all cats, and all cats were scored by the same person. Data from cats with a BCS other than 5 were not used for statistics on normal body weight. Means and standard deviations were calculated. A one-way ANOVA with breed as the classifying factor (only breeds with $n>6$ ), where breeds were sorted into

Abbreviation: BCS, body condition score.

*Corresponding author: Professor E. Kienzle, fax +49 892180 78702, email kienzle@tiph.vetmed.uni-muenchen.de 
homogeneous classes, was carried out using WinSTAT 2007.1 (WinSTATR, Fitch Software, Bad Krozingen, Germany). Breed differences in the incidence of overweight were compared by the $\chi^{2}$ test $(P<0.05)$. In addition, in forty cats, feline BMI according to Hawthorne \& Butterwick ${ }^{(10)}$ was applied as a control of BCS assessment, which agreed very well with short-haired cats (18-24\% body fat, according to feline BMI, in fifteen cats with a BCS of 5).

\section{Results}

The mean weight of lean cats (BCS 5) is given in Table 1. There were no significant differences between lean intact and neutered cats, except for male Maine Coons, where the intact males had an average weight of $6 \cdot 3$ (SD 1.1) $\mathrm{kg}(n$ 23) and the neutered males had 4.9 (SD 0.8) kg ( $n$ 5; $P<0.005)$. Within the males and females, five classes were identified, respectively. For female cats, the mean weights in these groups were as follows: very light $(2.8 \mathrm{~kg})$; light $(3.2 \mathrm{~kg})$; medium $(3.5 \mathrm{~kg})$; large $(4.0 \mathrm{~kg})$; giant $(4.9 \mathrm{~kg})$. For male cats, the corresponding values were $3 \cdot 6,4 \cdot 2,4 \cdot 3,5 \cdot 1$ and $6 \cdot 1 \mathrm{~kg}$. Males and females of the same breed did not always belong to the same class. In some breeds, individuals of the same sex were found in two different classes. The European Shorthair Cats of both sexes without a pedigree were classified as either light or medium.

The percentage of intact overweight cats (i.e. BCS > 5) was very low, it amounted to $7 \%$ of all intact males and $3 \%$ of all intact females (total number of intact males 192 and of intact females 247). By contrast, the incidence of overweight in neutered cats was $45 \%$. It amounted to $50 \%$ in neutered males ( $n$ 109), and to $38 \%$ in females ( $n$ 66). Among pedigreed cats, there were significant differences in the incidence of overweight $(\mathrm{BCS}>5)$ : Norwegian Forest Cats from cat shows had an incidence of overweight of $75 \%$ in neutered males and $50 \%$ in neutered females compared with 25 and $1 \%$ in Siamese/Oriental Shorthair Cats shown at cat shows. In pet cats without a pedigree, $51 \%$ of neutered males and $26 \%$ of neutered females were overweight. Cats with a BCS of 6,7 and 8 had on average $120 \%(n 71), 154 \%(n 23)$ and $214 \%$ ( $n$ 6) of the normal weight of their breed.

\section{Discussion}

Cats were weighed according to their availability in cat shows and in a small-animal practice. Obviously, this leads to an irregular number of cats weighed from different breeds and sexes. In principle, the population of cats from cat shows appears to be similar between breeds with regard to age (68-91\% of cats between 1 and 5 years of age) and a lower percentage of neutered cats (26\%). By contrast, cats without a pedigree showed a similar age distribution as described for cats in small-animal clinics $^{(11,12)}$ with only about $40 \%$ of the animals between 1 and 5 years of age. The percentage of neutered cats was $85 \%$. These differences between populations are unlikely to affect the body weight of cats grouped according to the BCS. However, the difference in age distribution probably affects the incidence of overweight and leanness, respectively. Overweight peaks in cat populations at the age of $4-6$ or 7 years of age ${ }^{(1,13)}$. Therefore, differences in the incidence of

Table 1. Body weight (BW) of male and female cats ${ }^{*}$ in ideal body condition scores (5) of different breeds

(Mean values, standard deviations and number of cats)

\begin{tabular}{|c|c|c|c|c|c|c|c|c|c|}
\hline \multirow[b]{3}{*}{ Breed $†$} & \multicolumn{5}{|c|}{ Males } & \multicolumn{4}{|c|}{ Females } \\
\hline & \multicolumn{2}{|c|}{$\mathrm{BW}(\mathrm{kg})$} & \multirow[b]{2}{*}{$n$} & \multirow[b]{2}{*}{ Class $\ddagger$} & \multirow[b]{2}{*}{ Breed } & \multicolumn{2}{|c|}{ BW (kg) } & \multirow[b]{2}{*}{$n$} & \multirow[b]{2}{*}{ Class* } \\
\hline & Mean & SD & & & & Mean & SD & & \\
\hline Scottish Fold & 3.0 & 0.78 & 3 & - & Abyssinian & $2 \cdot 8$ & 0.37 & 9 & Very light \\
\hline Siamese/Oriental Shorthair & $3 \cdot 4$ & 0.58 & 32 & Very light & Devon Rex & \multicolumn{2}{|c|}{$2.8 / 2.9$} & 2 & - \\
\hline Devon Rex & 3.4 & 0.98 & 4 & - & Korat & \multicolumn{2}{|c|}{$2 \cdot 6 / 3 \cdot 0$} & 2 & - \\
\hline Thai & \multicolumn{2}{|c|}{$3 \cdot 0 / 4 \cdot 1$} & 2 & - & Russian Blue & 2.9 & 0.38 & 16 & Very light \\
\hline Korat & \multicolumn{2}{|c|}{$3 \cdot 3 / 4 \cdot 0$} & 2 & - & Siamese/Oriental Shorthair & 2.9 & 0.39 & 15 & Very light \\
\hline Singapura & \multicolumn{2}{|c|}{3.8} & 1 & - & Exotic Shorthair & 2.9 & 0.45 & 5 & Very light \\
\hline Exotic Shorthair & $4 \cdot 0$ & 0.69 & 12 & Very light/light & Somali & $3 \cdot 1$ & 0.48 & 6 & - \\
\hline Balinese & \multicolumn{2}{|c|}{4} & 1 & - & Persian & $3 \cdot 1$ & 0.55 & 29 & Very light/light \\
\hline Abyssinian & $4 \cdot 1$ & 0.63 & 5 & - & Colourpoint & $3 \cdot 2$ & 0.26 & 3 & - \\
\hline Persian & $4 \cdot 1$ & 0.74 & 25 & Light & European Shorthair, no pedigree & $3 \cdot 4$ & 0.55 & 21 & Light/medium \\
\hline Bengal & $4 \cdot 2$ & 0.76 & 3 & - & Thai & 3.4 & 0.24 & 4 & - \\
\hline Russian Blue & $4 \cdot 2$ & 0.33 & 8 & Light/medium & Chartreux & \multicolumn{2}{|c|}{$3 \cdot 2 / 3 \cdot 7$} & 2 & \\
\hline Somali & $4 \cdot 2$ & 0.64 & 6 & - & Sacred Birman & 3.5 & 0.87 & 36 & Medium \\
\hline Burma & \multicolumn{2}{|c|}{$4 \cdot 3$} & 1 & - & Burma & \multicolumn{2}{|c|}{$3 \cdot 4 / 3 \cdot 6$} & 2 & - \\
\hline Sacred Birman & $4 \cdot 3$ & 0.59 & 20 & Light/medium & British Shorthair & 3.6 & 0.83 & 10 & Medium \\
\hline European Shorthair, no pedigree & $4 \cdot 3$ & 0.77 & 20 & Light/medium & Norwegian Forest Cat & 3.9 & 0.56 & 34 & Large \\
\hline Ragdoll & $4 \cdot 8$ & 0.38 & 6 & & Scottish Fold & $3 \cdot 9$ & 0.82 & 4 & - \\
\hline British Shorthair & $5 \cdot 1$ & 0.65 & 13 & Large & Selkirk Rex & \multicolumn{2}{|c|}{$5 \cdot 0 / 3 \cdot 0$} & 2 & - \\
\hline Norwegian Forest Cat & $5 \cdot 1$ & 0.71 & 22 & Large & Siberian Cat & $4 \cdot 1$ & 0.83 & 8 & Large \\
\hline Siberian Cat & $5 \cdot 3$ & 0.46 & 7 & Large & Ragdoll & $4 \cdot 2$ & $1 \cdot 26$ & 7 & Large \\
\hline Chartreux & \multicolumn{2}{|c|}{$5 \cdot 6$} & 1 & - & Maine Coon & $4 \cdot 8$ & 1.0 & 22 & Giant \\
\hline Maine Coon & $6 \cdot 1$ & $1 \cdot 2$ & 28 & Giant & & & & & \\
\hline
\end{tabular}

* Both intact and neutered cats in ideal body condition.

† Pedigreed, unless otherwise indicated.

$\ddagger$ Only classified if more than six individuals. 
overweight between the cats from cat shows and pet cats from a veterinary practice cannot be compared directly. If, however, the incidence of overweight is even higher in the younger cats from cat shows than in the more middle-aged group from veterinary practice, this can be interpreted as an even stronger suggestion of a difference in susceptibility to overweight. Thus, the high incidence of overweight in neutered Norwegian Forest Cats in comparison with cats without a pedigree but also in comparison with Siamese and Oriental Shorthair cats is remarkable. The differences are sufficiently large to suggest a breed disposition to overweight and leanness. It is, however, important to point out the role of the owners' aesthetic perception, which may affect their feeding behaviour, and thus eventually the incidence of overweight. Leanness may be more desirable in a very light elegant breed, and overweight more acceptable to the owners in a breed which is perceived as a big cat. Neutering had no effect on the body weight of lean cats, except in male Maine Coons, where intact males were heavier than neutered ones. It appears likely that this is an effect of selection for size, i.e. larger males are kept for breeding and smaller ones are neutered.

Cats with an average overweight of $214 \%$ were scored as BCS 8. The explanation is probably that (1) the study was focused on finding and weighing pure-bred cats in ideal body condition and (2) scoring was done together with the owner. It is quite possible that the reason why the fattest cats were scored 8 and not 9 is just psychological and subjective, i.e. this being more polite to the owners. It is remarkable that this happened in a scientific study, just a little outside the main purpose of the study.

In all breeds, there was a marked sexual dimorphism with heavier males than females. Even though this was more marked in some breeds than others, the difference is too big to allow a calculation of the mean of a group of cats regardless of their sex. Therefore, scientific papers, especially on weightloss experiments, should give the mean of the male and female cats separately. It is also not feasible to work with the average of $4 \mathrm{~kg}$ in a clinical setting or on nutrition guidelines, because it will overestimate a large percentage of the female feline population (given that a majority of the population has no pedigree). A female cat of $4 \mathrm{~kg}$ body weight from a very light, light or medium breed is likely to be between 11 and 43\% overweight. Therefore, at least for the standard non-pedigreed European Shorthair cats, a different ideal weight or ideal weight range for females and males should be used.

\section{Acknowledgements}

The authors thank all cat owners who cooperated in the study. There is no conflict of interest. In present study received no specific grant from any funding agency in the public, commercial or not-for-profit sectors. K. M. obtained the data and statistically evaluated the data. E. K., in part, statistically evaluated the data and wrote the manuscript.

\section{References}

1. Russel K, Sabin R, Holt S, et al. (2000) Influence of feeding regimen on body condition in the cat. J Small Anim Pract 41, 12-17.

2. Villaverde C, Ramsey JJ, Green AS, et al. (2008) Energy restriction results in a mass-adjusted decrease in energy expenditure in cats that is maintained after weight regain. J Nutr 138, 856-860.

3. Strasser H (1968) Über Aufbau und Fortführung einer Katzenzucht (Establishment and maintenance of a cat colony). Zeitschrift für Versuchstierkunde 10, 137-146.

4. Loveridge GG \& Rivers JPW (1989) Body weight changes and energy intakes of cats during pregnancy and lactation. In Nutrition of the Dog and Cat, [IH Burger and JPW Rivers, editors]. Cambridge: Cambridge University Press.

5. Hendriks WH \& Wamberg S (2000) Milk intake of suckling kittens remains relatively constant from one to four weeks of age. J Nutr 130, 77-82.

6. Stratmann B (1988) Untersuchungen zur Körperzusammensetzung von Katzen (Investigations on body composition of cats). Doctoral Thesis, Hannover: Tierärztliche Hochschule.

7. Hendriks WH, Moughan PJ \& Tarttelin MF (1997) Body composition of the adult domestic cat (Felis catus). J Anim Physiol Anim Nutr 77, 16-23.

8. Lauten SD, Cox NR, Baker GH, et al. (2000) Body composition of growing and adult cats as measured by use of dual energy X-ray absorptiometry. Comp Med 5, 175-183.

9. Laflamme DP (1997) Development and validation of a body condition score system for cats: a clinical tool. Feline Pract 25, 13-18.

10. Hawthorne A \& Butterwick RB (2000) Predicting the body composition of cats: development of a zoometric measurement for estimation of percentage body fat in cats. $J$ Vet Intern Med 14, 365.

11. Kraft W (2003) Geriatrie bei Hund und Katze (Geriatrics in the Dog and Cat), 2nd ed. Stuttgart: Parey Verlag.

12. Davies M (1996) Canine and Feline Geriatrics. Oxford: Blackwell Science.

13. Scarlett M, Donoghue S, Saidla J, et al. (1994) Overweight cats: prevalence and risk factors. Int J Obes Relat Metab Disord 18, 22-28. 